\title{
A high-brightness accelerator-based EUV source for metrology applications
}

\author{
Yasin Ekinci, Terence Garvey, Andreas Streun, Albin Wrulich, and Leonid Rivkin \\ Paul Scherrer Institute, 5232 Villigen PSI, Switzerland \\ Contact: yasin.ekinci@psi.ch
}

\begin{abstract}
One of the challenges of actinic metrology tools for EUV lithography is the availability of light sources with high brightness, stability, and availability. In particular, actinic patterned mask inspection on EUV reticles is considered an essential tool for the EUV lithography ecosystem and it requires an EUV source of high brightness. We present the design of a compact and accelerator-based light source producing EUV radiation with high-brightness for actinic metrology applications in the semiconductor industry. Our design is based on the well-established components and design principles. The specifications required for actinic mask inspection is achieved using a short period undulator and $430 \mathrm{MeV}$ electron energy. The concentric design of storage and booster rings enables stable operation with a relatively small footprint. This study shows the commercial viability of a compact and high-brightness EUV source with high stability and reliability and demonstrates its feasibility for actinic metrology applications.
\end{abstract}

Keywords: EUV, lithography, metrology, source, mask inspection, actinic

\section{INTRODUCTION}

EUV metrology sources with $13.5 \mathrm{~nm}(92 \mathrm{eV})$ wavelength are needed for EUV mask manufacturing at several stages, such as blank inspection, patterned mask inspection, mask review, final inspection, and pellicle inspection [1-4]. Future applications of such sources in a similar wavelength range may also involve applications other than EUV mask metrology, such as wafer inspection and mask substrate inspection [5]. In particular, actinic patterned mask inspection is considered an essential tool for the implementation of EUV lithography in high-volume manufacturing. One of the challenges in developing such a tool is the availability of a source delivering EUV light with high brightness, excellent stability, high availability and sufficient flux.

The required specifications for the source depend on the specific applications. The most important ones are high brightness, stability, and availability. For mask review, the brightness requirements are very low since the fast scanning of large areas is not needed. For blank inspection a brightness of $100 \mathrm{~W} / \mathrm{mm}^{2} \cdot \mathrm{sr}$ is sufficient. For patterned mask inspection, a brightness of $300 \mathrm{~W} / \mathrm{mm}^{2} \cdot \mathrm{sr}$ is probably sufficient based on simple estimations. However, for a more realistic case, the requirement increases significantly due to various issues such as the flare due to the roughness of the optics, mask multilayer and line edge roughness of the absorber pattern, detector noise, source fluctuations, limited spatial resolution, etc. Moreover, in an inspection of a $10 \times 10 \mathrm{~cm}^{2}$ area with nanoscale resolution, the image map reaches to a size that is on the order of Terapixels and the signal-to-noise ratio should be above 8 in order to keep the number of false positives reasonably low. Therefore, the required brightness for actinic patterned mask inspection is higher. For example, according to a paper from Sematech and Intel [6], one inspection tool supplier estimated the required source brightness to be $\sim 2,500 \mathrm{~W} / \mathrm{mm}^{2} \cdot \mathrm{sr}$. In addition, an important aspect of any tool or method in the semiconductor industry is the extendibility to the future technology nodes. With the advancement of nodes, feature size and printable defect size decrease, which in turn increase the required photon numbers to resolve and detect these with the metrology tool. Therefore, from an ideal EUV source we need $1,000-10,000 \mathrm{~W} / \mathrm{mm}^{2} \cdot \mathrm{sr}$ brightness for patterned mask inspection. Lensless imaging methods that rely on the coherence property of the light, despite being a relatively exotic method [7-9], are the only methods that have shown, in the past, promising results. They require a brightness that is several orders of magnitude higher than that of the incoherent microscopy techniques. The required brightness for this technique for actinic patterned mask inspection is about $\mathrm{GW} / \mathrm{mm}^{2} \cdot \mathrm{sr}$ and significantly lower for blank inspection or mask review.

Photomask Technology 2018, edited by Emily E. Gallagher, Jed H. Rankin, Proc. of SPIE Vol.

10810, 108100W · @ 2018 SPIE · CCC code: 0277-786X/18/\$18 · doi: 10.1117/12.2501930 
Conventional ways of generating EUV light are Z-pinch discharge plasma, LPP (laser produced plasma), and HHG (high harmonic generation). Z-pinch sources have high flux, high availability and need low maintenance, but come with a low brightness of about $10 \mathrm{~W} / \mathrm{mm}^{2} \cdot \mathrm{sr}$ [10]. For higher brightness, LPP sources are needed and these can provide a brightness of about $100-300 \mathrm{~W} / \mathrm{mm}^{2} \cdot \mathrm{sr}$ [11-13]. We note that a review of the literature shows also much higher brightness values and it is out of the scope of this paper to make an assessment on the status of the plasma sources. Further development will improve the stability and reliability of LPP sources. A very high brightness can be achieved with an HHG source but this time the flux becomes the bottleneck which is currently limited in the $\mathrm{nW}$ range [14]. Further development may bring this technology to the power levels of $\mu \mathrm{W}$. Moreover, for the aforementioned technologies debris-mitigation, outof-band radiation, stability, and reliability remain crucially challenging issues.

An alternative approach for the generation of EUV light would be to use accelerator -based technology. Synchrotron light generation is an elegant way of generating light with short wavelengths in a controlled manner. Therefore, there are many synchrotrons in operation in the world serving the needs of many research fields at different energies with different optical methods. Nevertheless, these sources are typically large-scale facilities and therefore not easy to install at industrial sites. To be compatible with industrial use, the source should be relatively compact, fitting into the existing infrastructure of a manufacturing environment.

Several compact synchrotrons were built in the 80's and 90's [15-16]. These tools were designed for lithography, not for metrology applications. However, the target specifications of the required flux in lithography evolved and, therefore, they were abandoned as they could provide a flux of only $\sim 10 \mathrm{~W}$. Moreover, they were based on the technology of 30 years ago and accelerator technology has since made significant progress. In addition, they were not really compact as the linac and booster injection systems were housed in an adjacent room and therefore the facility occupied a larger footprint than that of the compact storage ring alone.

Based on these facts, and recognizing the imminent need for better EUV metrology sources, we have designed a compact synchrotron source which can be used for EUV metrology applications in a manufacturing environment. Our design strategy has been as follows:

- Sufficient flux: Enabling one to perform all EUV metrology applications within reasonable time, i.e. a clean EUV power of $10-100 \mathrm{~mW}$.

- High brightness: A brightness that can cover the requirements of all EUV metrology applications and different imaging methods, in particular for the lensless imaging methods which rely on high coherence.

- Intensity stability: Extreme beam stability to avoid complications which may arise from the very short image acquisition times due to fast optical scanning.

- Compact design: As compact as possible, but without increasing the machine complexity or compromising the performance.

- Reliability: Enabling one to achieve minimal downtime by using proven technology and adopting robust design features.

- Minimize R\&D costs and lead time: Choosing only proven concepts and technologies, and commercially available components whenever possible.

In the following section, we describe the major components and characteristics of this source. We address the optics design of the storage ring as well as the main technological sub-systems for the accelerator, such as the linac injector, the radio-frequency systems, the vacuum design, transfer line optics, and undulator design. We present the radiation shielding concept, which ensures the safe operation and strict requirements of the regulatory agencies. The mechanical integration of the overall layout has been studied and the main systems engineering aspects have been addressed.

\section{DESIGN AND DISCUSSIONS}

We have designed a compact and accelerator-based EUV source for actinic metrology applications in the semiconductor industry. The main components are the so-called storage ring, the booster ring, and the undulator as seen in Figure 1. Our design is based on well-established components and design principles [18], minimizing the lead time of its realization and the technical risks to reach the target specifications. The novel design [19-20] of a concentric storage ring and 
booster ring enables top-up operation with a footprint of $5 \times 12 \mathrm{~m}^{2}$. This footprint is adopted to achieve a compact design without compromise on stability, reliability, availability, and lead time.

We have introduced innovative solutions compared to the sources developed in the nineties, which include:

$\rightarrow$ Adopt the technology of diffraction-limited storage rings

$\rightarrow$ Concentric design with vertical stacking of booster and storage rings, leading to a small footprint

$\rightarrow$ Multi-bend magnet lattice

$\rightarrow$ Implementation of undulator

$\rightarrow$ Compact, combined-function magnets

$\rightarrow$ Small vacuum chambers with NEG coating

$\rightarrow$ Photo-injector linac enabling a compact design

We have adopted the latest technologies developed for diffraction-limited storage rings which are the latest generation of synchrotrons [21]. This machine would operate in top-up mode, enabling always same current and thereby photon flux with $0.1 \%$ beam stability. This is achieved by using a full energy booster ring. The electron beam energy is $430 \mathrm{MeV}$ and the beam current is $150 \mathrm{~mA}$. We have 50 ps pulses with a $500 \mathrm{MHz}$ repetition rate. It provides about $100 \mathrm{~mW}$ with about $1 \mathrm{GW} / \mathrm{mm}^{2} \cdot$ sr brightness. Table 1 summarizes the major parameters of the source.

EUV light with a low emittance (about 10 and $2 \mathrm{~nm} \cdot \mathrm{rad}$ respectively along the horizontal and vertical axes) is generated in a 3.2-meter-long undulator of $16 \mathrm{~mm}$ period length installed in the electron storage ring of circumference $25.8 \mathrm{~m}$ with electron beam energy of $430 \mathrm{MeV}$. Our design is based essentially on mature, well-established accelerator system technologies which have been used routinely for many years for research purposes in national laboratories. This approach favors the requirement for high reliability. The magnet optics design of the storage ring is based on the novel techniques currently being applied to the design and construction of the advanced light sources, which allows us to reach a relatively small emittance, and thus high-brightness for a compact race-track ring. The layout of the accelerator complex, including a linear accelerator injector, a booster synchrotron, and the electron storage ring, has been designed to keep the footprint of the EUV source as small as is reasonably possible.

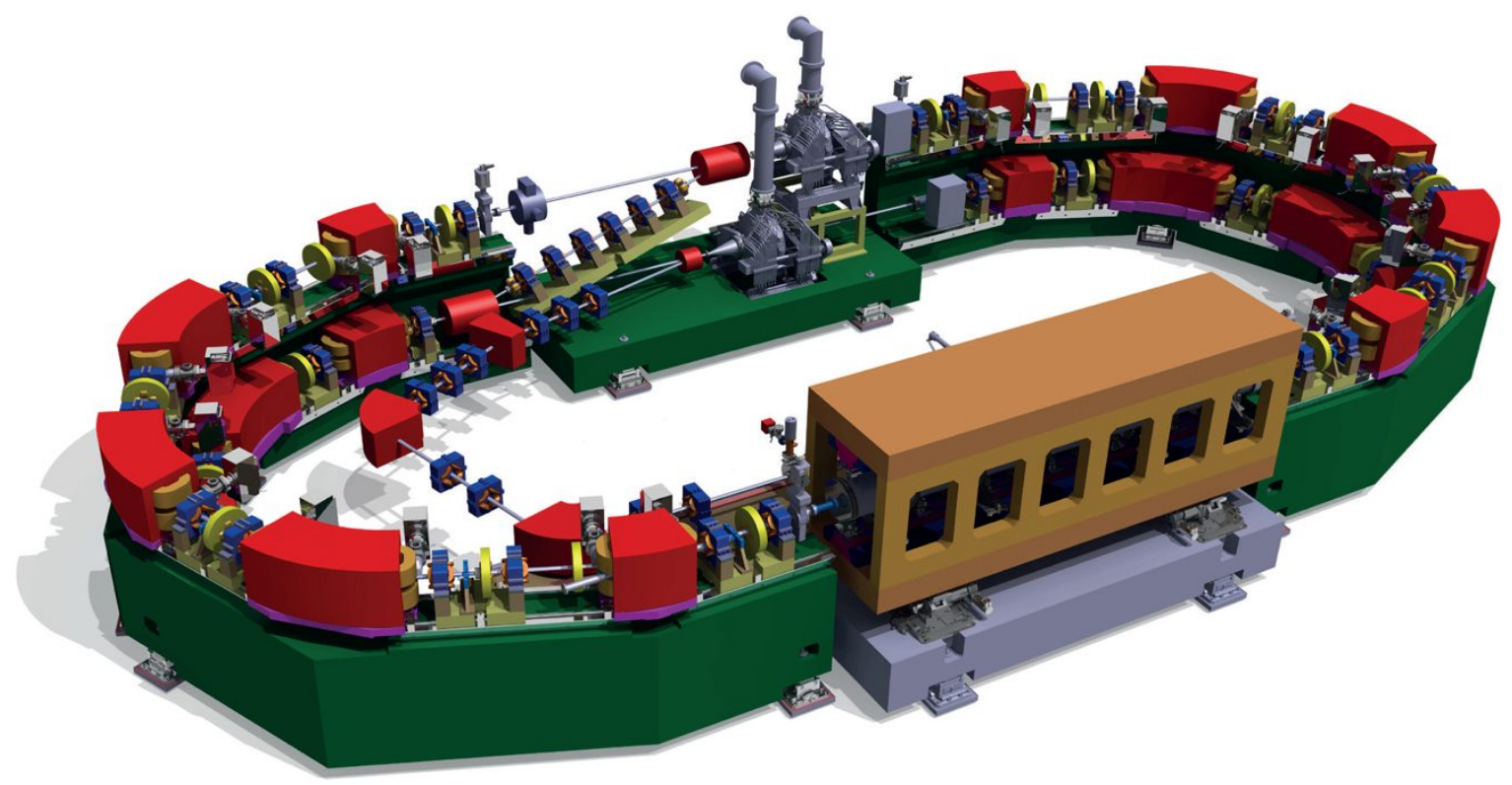

Figure 1.3D mechanical integration of COSAMI (COmpact Source for Actinic Mask Inspection). 
Table 1. Basic parameters of the source.
Wavelength
$13.5 \mathrm{~nm}$
Flux
$\sim 100 \mathrm{~mW}$
Brilliance
$\sim 10^{9} \mathrm{~W} /\left(\mathrm{mm}^{2} \cdot \mathrm{sr}\right)$
Bandwidth
$0.5 \%$
Beam energy/beam current
$430 \mathrm{MeV} / 150 \mathrm{~mA}$
Pulse structure
$\sim 50$ ps every 2 ns
Beam stability
$0.1 \%$
Availability
$>95 \%$
Reliability
$<1 \%$ downtime
Footprint
$5 \mathrm{~m} \times 12 \mathrm{~m}$

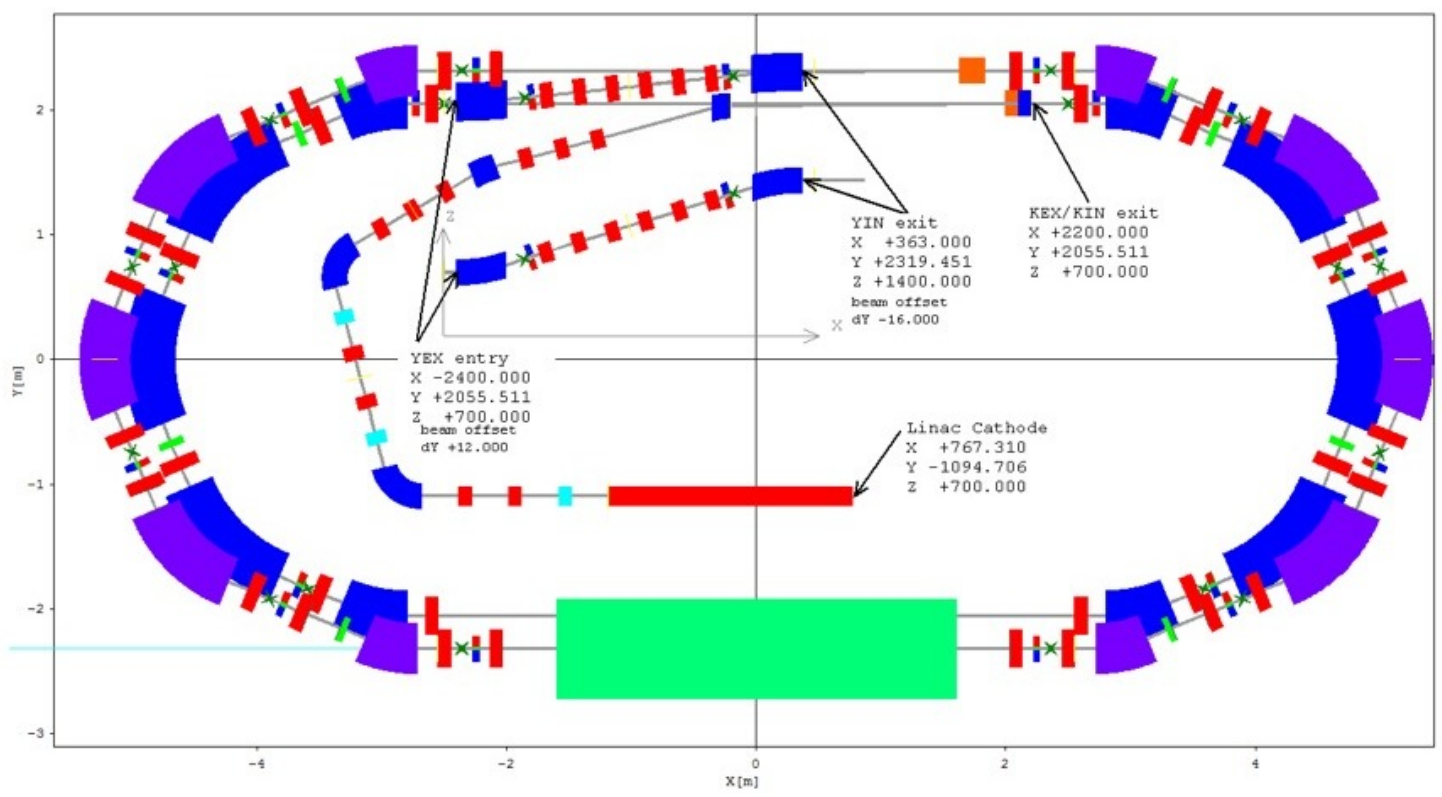

Figure 2. The schematic layout of the linac, booster, and storage ring. 


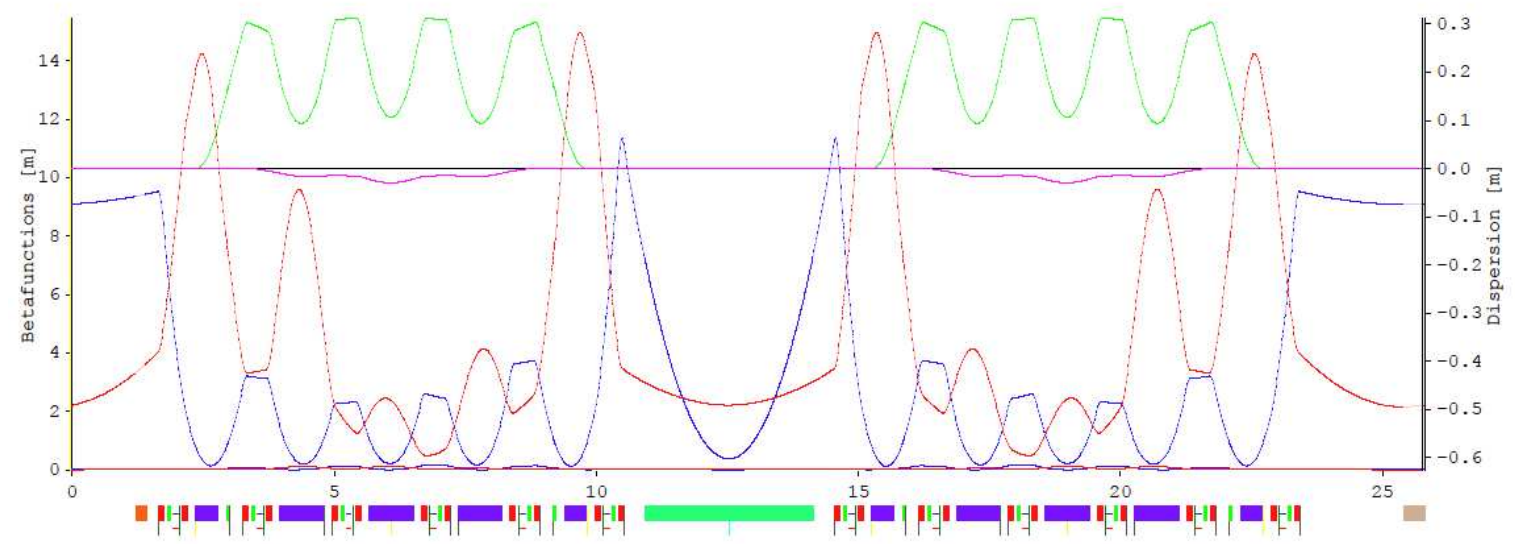

Figure 3. Storage ring optical functions $\left(\beta_{x}, \beta_{y}, D_{x}, D_{y}\right)$. Bending magnet positions are indicated in blue. The undulator is represented by the green bar.

Figure 2 shows the schematic of the optical lattice. The electrons are generated on a cathode and accelerated to $43 \mathrm{MeV}$ using a linac of 2.1 meters. They are then injected into the booster ring with a circumference of 24 meters where the electrons are further accelerated to $430 \mathrm{MeV}$, ready for injection into the storage ring where they circulate at constant energy. The total circumference is 25.8 meters with two straights and two 5-bend achromat arcs. One straight section is for the undulator and the other one is for injection into the storage ring and RF cavity unit. The electrons pass through the undulator and generate EUV light.

Figure 3 shows the optical function along the different positions of the storage ring. The horizontal beta-function is almost periodic in the arcs with small values in the bending magnets to generate a low emittance. It is minimized by optimum matching to the diffraction phase-space in the undulator to achieve high coherence. It is large in the injection straight to allow for a maximum offset of the injected beam. The vertical beta function is minimized at the undulator edges, taking into account the undulator's vertical focusing, which is not negligible at this low energy.

The resulting parameters of the storage ring design are listed in Table 2. The storage ring circumference corresponds to 43 RF wavelengths. 24 "buckets" are filled to leave a gap in the bunch train to combat ion trapping. The total charge in the ring is $\sim 17 \mathrm{nC}$ (assuming a beam current of $200 \mathrm{~mA}$, to have some margin). Non-linear beam dynamic studies were performed to evaluate the dynamic aperture, Touschek scattering, and intra-beam scattering effects have been analyzed. The beam lifetime due to scattering is 15 mins. This requires frequent "top-up" injections in order to reach $0.1 \%$ beam stability.

Table 2. Basic parameters of the storage ring.

\begin{tabular}{|l|c|l|r|}
\hline Circumference $[\mathrm{m}]$ & 25.8 & Energy [MeV] & 430 \\
\hline Working Point $\mathrm{Q}_{\mathrm{x} / \mathrm{y}}$ & $4.73 / 1.58$ & Radiation loss/turn [keV] & 2.83 \\
\hline Natural chromaticity $\xi_{\mathrm{x} / \mathrm{y}}$ & $-9.7 /-6.9$ & Emittance $[\mathrm{nm}]$ & 5.50 \\
\hline Momentum compaction $\alpha_{\mathrm{c}}$ & 0.0258 & Relative energy spread & $4.13 \cdot 10^{-4}$ \\
\hline Hor. damping partition $\mathrm{J}_{\mathrm{x}}$ & 1.54 & Damping times $\tau_{\mathrm{x} / \mathrm{y} / \mathrm{E}}[\mathrm{ms}$ ] & $16.6 / 25.6 / 17.5$ \\
\hline
\end{tabular}


a)

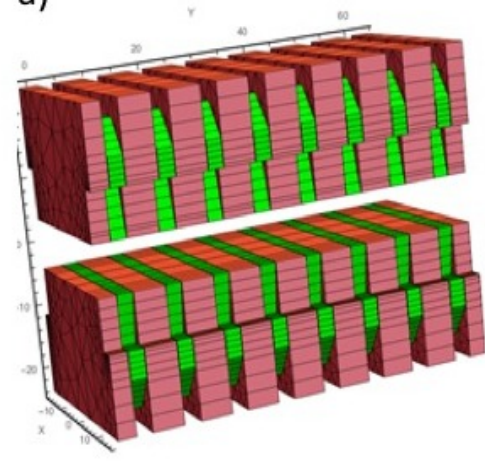

b)

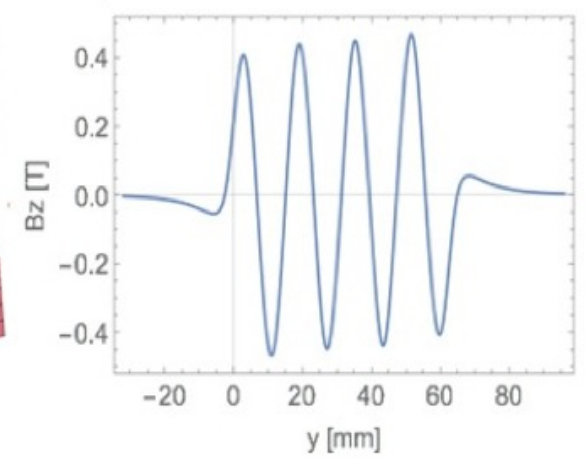

Figure 4. (a) The magnet structure of the undulator. (b) RADIA simulation of 4 periods of the magnet structure showing the on-axis magnetic fields. The entire undulator has 200 periods.

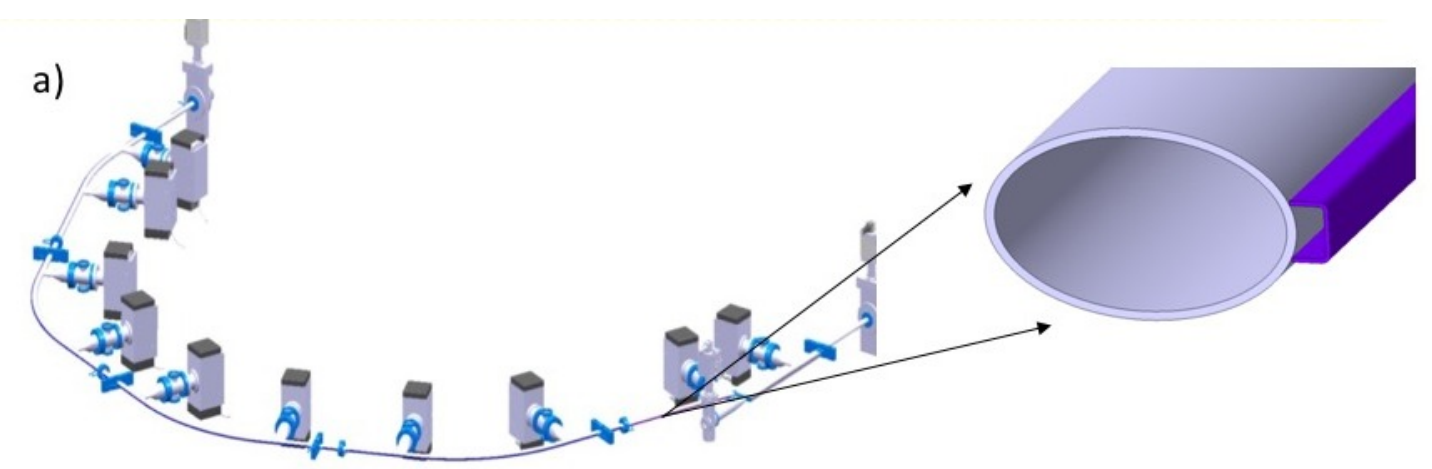

b)

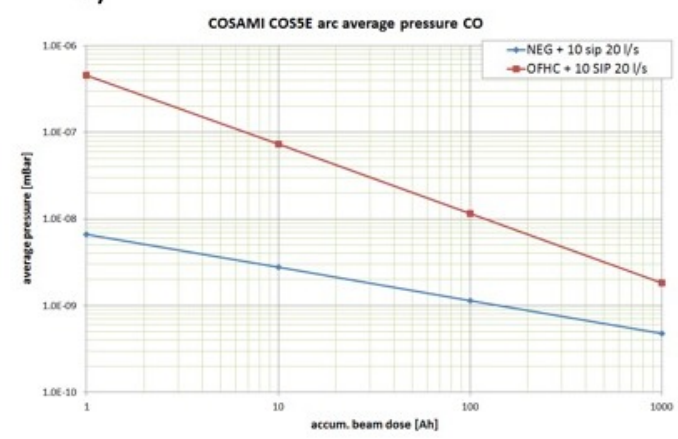

c)

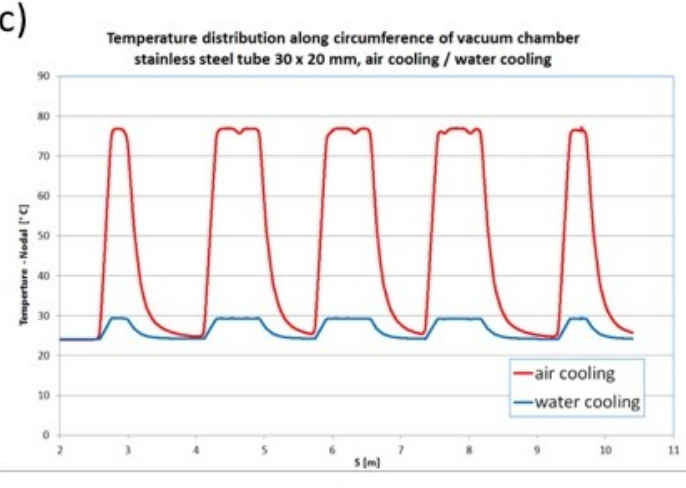

Figure 5. (a) Vacuum chamber layout for one magnet arc. Close-up layout: Vacuum tube cross-section and cooling channel (b) Comparison of vacuum chamber pressure with and without NEG coating. (c) Temperature distribution in a stainless steel dipole chamber with air and water cooling.

The undulator design is shown schematically in Figure 4. An undulator is a series of magnets with alternating poles producing an alternating vertical (w.r.t. the orbit plane) magnetic field. When electrons pass through the undulator, they "wiggle" horizontally and this transverse oscillation of electrons generates radiation. We have specified the undulator parameters to have a $0.4 \mathrm{~T}$ magnetic field and a periodicity of $16 \mathrm{~mm}, 7 \mathrm{~mm}$ gap spacing, and a length corresponding to 200 periods. Simulations of the magnetic field (Figure 4(b)) shows that a uniform magnetic field of $0.4 \mathrm{~T}$ is achievable 
within a region of $\pm 12 \mathrm{~mm}$. The undulator with two hundred periods provides EUV light with a bandwidth of 1/200= $0.5 \%$. We fixed the undulator period to $16 \mathrm{~mm}$, because this short periodicity has already been shown to be possible at SwissFEL. Undulators of shorter periods may introduce certain complications or risks. We have chosen NdFeB, treated with diffusion of Dysprosium, for the magnets. At room temperature, this material provides the best combination of remanent field and coercivity. A high coercivity prevents magnets from demagnetization due to beam loss.

An extremely good vacuum is required since residual gas leads to scattering and loss of electrons. The vacuum chamber (partly shown in Figure 5(a)) has an elliptical shape with $3 \times 2 \mathrm{~cm}^{2}$ dimensions. Maintaining a good vacuum $\left(<10^{-9} \mathrm{mbar}\right)$ in such a long and narrow chamber is challenging. The base pressure is dominated by photo-desorption due to synchrotron radiation. At certain intervals, ion pumps are installed. Nevertheless, this is still not sufficient and further improvement is achieved by using NEG coating, which acts as a getter surface. The comparison of vacuum pressure with and without the NEG coating is shown in Figure 5(b).

The synchrotron radiation heat load is low, but it is still $85 \mathrm{~W} / \mathrm{m}$ which increases the temperature by 80 degrees and this will substantially increase the vacuum pressure. Therefore cooling is needed and this is achieved by water cooling pipe attached to the whole ring as shown in Figure 5(a). The comparison of the temperature with and without water cooling is shown in Figure 5(c).

Figure 6 shows examples of hardware for some of the sub-systems. We adopted the photo-injector electron source employed in short wavelength X-ray FELs. It is a traveling wave gun with a 2.1-m-long C-band structure. It is very compact compared to the conventional thermionic gun based linac systems. The electrons are generated by a UV-Laser system which provides photo-emitted electrons when the laser beam strikes a semiconductor cathode. Electrons lose about $500 \mathrm{~W}$ when they pass through the storage ring dipoles and this loss is compensated by the RF cavity. The cavity is powered by a solid state amplifier. Such amplifiers have already been developed by SOLEIL and by PSI and are commercially available.

For the installation of such a tool, radiation safety is a big concern and specific precautions have to be taken for safety reasons. The room has to be isolated and interlocked. Radiation dose simulations were performed using the Monte Carlo particle transport codes (MCNPX 2.7.0 and MCNP 6.1). The results are shown in Figure 7. The top and side walls are made of 20-cm-thick standard concrete, 7 -cm-thick lead layer, 15 -cm-thick lead plugs, and a 25 -cm-thick polyethylene layer. An additional polyethylene layer is placed around the undulator and the collimator, where higher radiation rates are predicted. It is concluded that the radiation standards of international and local regulatory agencies can be met, allowing people to work outside of the walls without special precautions. This design needs further optimization in order to reduce the wall thickness by installing local shielding as close as possible to the points where the radiation rates are higher.

(a)

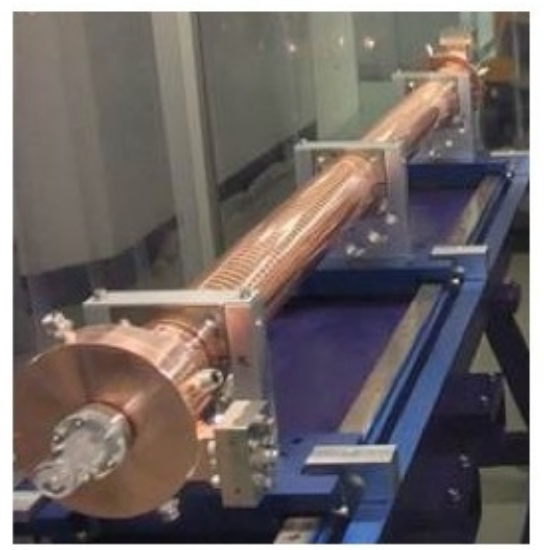

(b)

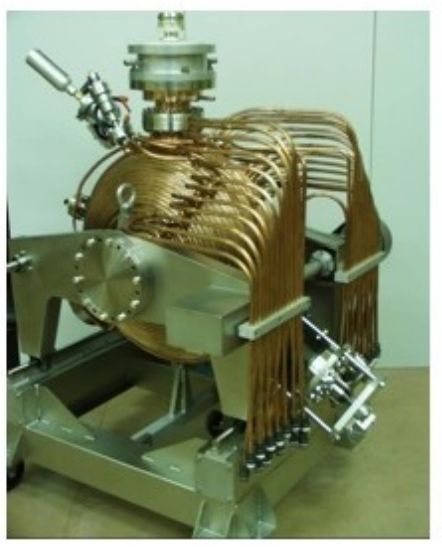

(c)

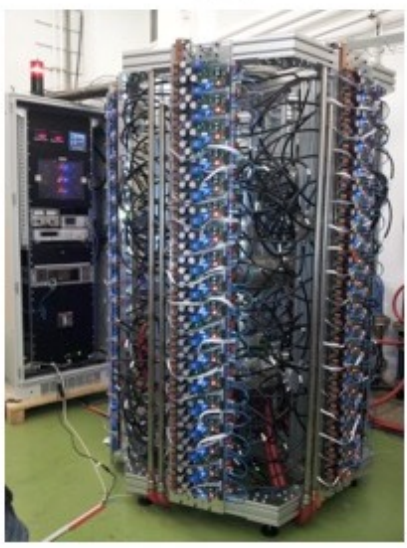

Figure 6. Pictures of (a) traveling wave structure, (b) RF cavity, and (c) solid-state amplifier. 

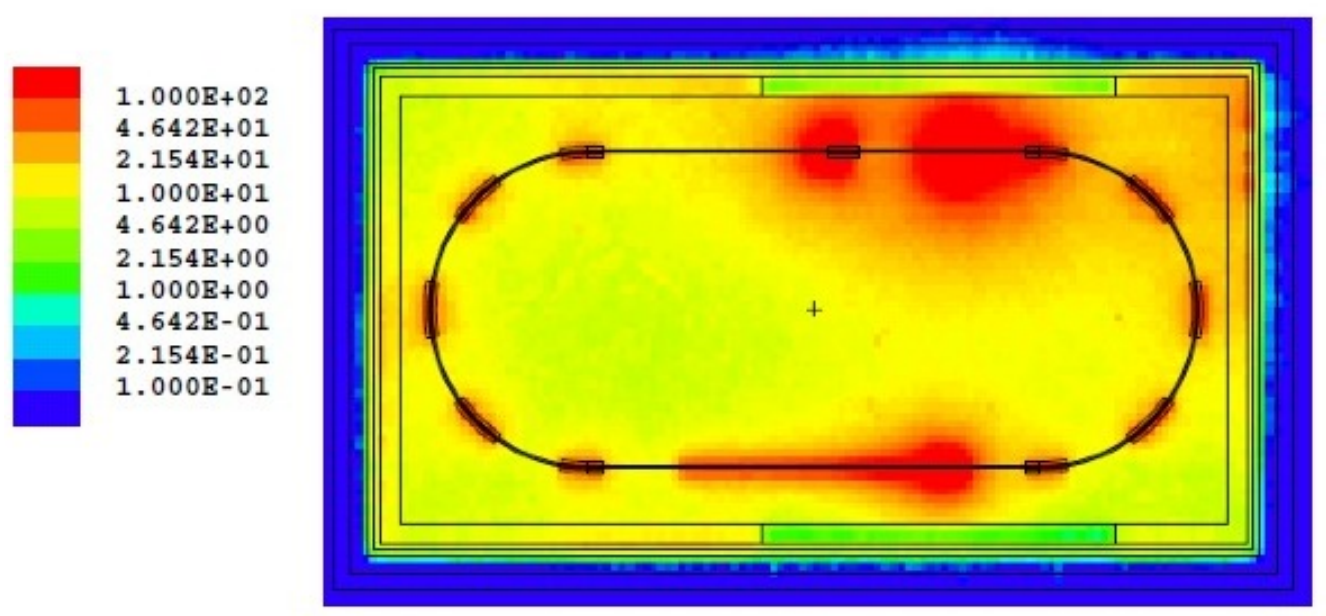

\section{Top view}

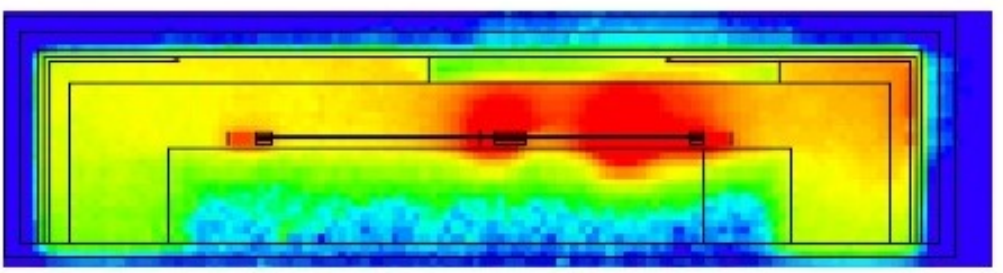

\section{Side view}

Figure 7. Radiation distribution in the facility. The units are in $\mu \mathrm{SV} / \mathrm{h}$.

\section{SUMMARY AND CONCLUSIONS}

In summary, we have completed the optical design of an accelerator-based EUV source via extensive simulations, optimized the design for minimal emittance, and identified technical solutions for all of the major sub-systems. Our aim was to design a source which can cover all EUV metrology applications and methods with the required stability, maximum brightness, and sufficient flux. High reliability and minimum maintenance were aimed at as the source will be employed in a production environment. Our philosophy was to adopt proven and well-established technologies in order to reach a short delivery time by avoiding R\&D and prototyping. For a fabrication environment, a compact design, without compromising performance, was an objective of our design. In a fab, the tool would be installed in the sub-floor and the light would be transferred to the cleanroom floor with a delivery optics serving as a source for one or a few applications. The footprint is similar to the laser system of an EUV scanner and ensuring the radiation safety standards can be easily met. In conclusion, COSAMI is a viable solution for the EUV metrology.

\section{ACKNOWLEDGMENTS}

The authors are grateful to M. Aiba. R.M. Bergmann, T. Bieri, M. Calvi, P. Craievich, M. Ehrlichman, C. Gough, P. Lerch, A. Mueller, M. Negrazus, C. Rosenberg, T. Schmidt, L. Schulz, L. Stingelin, V. Vrankovic, A. Zandonella Gallagher, and R. Zennaro of PSI and U. Klein and J. Rehanek of Advanced Accelerator Technologies (AAT) for their contributions and discussions. This project has received partial funding from the Swiss Commission for Technology and Innovation under grant \# 19193.1PFNM-NM.

\section{REFERENCES}

[1] B. Turkot, S. L. Carson, A. Lio, T. Liang, M. Phillips, B. McCool, E. Stenehjem, T. Crimmins, G. Zhang, and S. Sivakumar, "EUV Progress Toward HVM Readiness,” Proc. SPIE 9776, 977602 (2016)

[2] T. Suzuki, H. Miyai, K. Takehisa, H. Kusunose, H. Watanabe, and I. Mori, "Detection capability of Actinic Blank Inspection tool," Proc. SPIE 9658, 965800 (2015) 
[3] R. Hirano, S. Iida, T. Amano, H. Watanabe, M. Hatakeyama, T. Murakami, S. Yoshikawa, K. Suematsu, and K. Terao, "Study of extreme ultraviolet lithography patterned mask inspection tool for half-pitch 11-nm node defect detection performance," J. Micro/Nanolith. MEMS MOEMS 15, 021008 (2016)

[4] D. Hellweg, J. Ruoff, A. Herkommer, J. Stühler, T. Ihl, H. Feldmann, M. Ringel, U. Strößner, S. Perlitz, and W. Harnisch, "AIMS EUV: the actinic aerial image review platform for EUV masks," Proc. SPIE 7969, 79690H (2011)

[5] F. Brizuela, G. Vaschenko, C. Brewer, M. Grisham, C. S. Menoni, M. C. Marconi, J. J. Rocca, W. Chao, J. A. Liddle, E. H. Anderson, D. T. Attwood, A. V. Vinogradov, I. A. Artioukov, Y. P. Pershyn, and V. V. Kondratenko, "Reflection mode imaging with nanoscale resolution using a compact extreme ultraviolet laser," Opt. Express 13, 3983 (2005)

[6] Y. D. Chan, A. Rastegar, H. Yun, E. S. Putna, and S. Wurm, "EUV mask defect inspection and defect review strategies for EUV pilot line and high volume manufacturing," Proc. SPIE 7636, 76361D (2010)

[7] I. Mochi, P. Helfenstein, I. Mohacsi, R. Rajeev, D. Kazazis, S. Yoshitake, and Y. Ekinci, "RESCAN: an actinic lensless microscope for defect inspection of EUV reticles," J. Micro/Nanolith. MEMS MOEMS 16, 041003 (2017)

[8] P. Helfenstein, I. Mochi, R. Rajeev, S. Fernandez, and Y. Ekinci, "Coherent Diffractive Imaging Methods for Semiconductor Manufacturing," Adv. Opt. Technol. 6, 439 (2017)

[9] I. Mochi, R. Rajeev, P. Helfenstein, S. Fernandez, D. Kazazis, and Y. Ekinci, "Through-pellicle inspection of EUV masks," Proc. SPIE 10583, 105831I (2018)

[10] M. J. Partlow, M. M. Besen, P. A. Blackborow, R. Collins, D. Gustafson, S. F. Horne, and D. K. Smith, "Extreme ultraviolet light source development to enable pre-production mask inspection," J. Micro/Nanolith. MEMS MOEMS 11, 021105 (2012)

[11] A. Z. Giovannini, O. Morris, I. Henderson, S. Ellwi, and R. S. Abhari, "EUV brightness, spot size, and contamination measurements at the intermediate focus," Proc. SPIE 7969, 79692X (2011)

[12] A. Vinokhodov,1 M. Krivokorytov, Yu. Sidelnikov, V. Krivtsun, V. Medvedev, V. Bushuev, K. Koshelev, D. Glushkov, and S. Ellwi, "Stable droplet generator for a high brightness laser produced plasma extreme ultraviolet source,” Rev. Sci. Inst. 87, 103304 (2016)

[13] Imam Kambali, Enda Scally, Padraig Dunne, Gerry O'Sullivan, Paul Sheridan, Emma Sokell and Fergal O'Reilly, "A tin-gold alloy based EUV source for metrology applications," J. Phys. D: Appl. Phys. 46, 495104 (2013)

[14] X. Zhang, J. Garlick, E. Mountfort, H. Kapteyn, "A tabletop coherent EUV source for commercial EUVL metrology and imaging applications," Proc. SPIE 10583, 105831C (2018)

[15] T. Miyatake, X. Li, S. Hirose, T. Monzen, K. Fujii, and K. Suzuki, "Compact synchrotron radiation lithography system for $70 \mathrm{~nm}$ device manufacturing,” J. Vac. Sci. Technol. B 19, 2444 (2001)

[16] J. R. Maldonado, "Overview of X-ray lithography at IBM using a compact storage ring," Conference Record of the 1991 IEEE Particle Accelerator Conference, pp. 542-546 (1991)

[17] D. C. Ockwell, N. C. E. Crosland, and V. C. Kempson, "Synchrotron light as a source for extreme ultraviolet lithography," J. Vac. Sci. Techol. B 17, 3043 (1999)

[18] T. Garvey, Y. Ekinci, L. Rivkin, A. Streun, and A. Wrulich, "A compact high-brightness accelerator-based EUV source for actinic mask inspection ," OSA Technical Digest (online), High-Brightness Sources and Light-driven Interactions, paper ET3B.5.

[19] A. Wrulich, Y. Ekinci, A. Streun, and L. Rivkin, "A compact light source for metrology applications in the EUV range," 2015P17100EP

[20] T. Garvey, L. Rivkin, A. Streun, A. Wrulich, and Y. Ekinci, “A compact storage ring for the production of EUV radiation," Proceedings of ANS AccApp'17: 13th Int. topical meeting on the applications of accelerators (2017)

[21] P.F. Tavares, S.C. Leemann, M. Sjöström, and Å. Andersson, “The Max IV storage ring project”, J. Synchrot. Rad. 21, 862-877 (2014) 